des disjoncteurs. Ce transformateur est à isolement à huile el à refroidissement par l'air ; il est disposé sous la voiture.

Entre l'archet de prise de courant et l'auto-transformateur, (st intercalé un commutateur qui permet de relier cet archet, soit à la première borne de l'auto-transformateur, lorsqu'on ulilise le courant à Io ooo volts, soit directement à une prise spéciale de ce transformateur, correspondant à une tension de too volts, lorsqu'on se lrouve à l'inléricur de Limoges.

Ce commulateur est enfermé dans une armoire en tôle, situéc au milieu de chaque automotrice. Cette armoirc contient en outre des fusibles à haute et basse tension, un disjoncteur à maxima inséré dans le circuit des moteurs, el un disrupleur à plaque de mica, cestiné à mettre à la terre le circuit à basse tension au cas où celui-ci viendrait à être mis fortuitement en contact avec la haute tension. En dehors de cotte armoire, un limiteur de tension ct un parafoudre i cornes complètent la protection de la voiture.

Les freins sont à air, du système Houplain. Ils forction aussi bien sur les petites lignes de chemins de f'ex d'intérèt local que sur les grandes lignes de chemins de fer d'intérêt général. Aussi, avons-nous pensé qu'il serait sans doule intéressant pour les lecteurs de La Houlle Blanche de methe sous leurs yeux la description des installations nécessaires i la traction électrique des trains des Chemins de fer départementaux de la Haule-Vicme.

P. Chazeaux, Ingénieur-Electricien.

\section{USINE HYDRO-ÉLECTRIQUE de I'EAST CANADA CREEK}

I a Easl Creel: Lighl di Power Company vient de terminer l'installation d'une usine hydro-électrique à Inghams Mills, dans l'Etat de New-York, sur l'East Canada Creek, à ro kilomètres de la ville de Litule Falls, et à 8 kilomètres en amont du point où ce cours d'eau

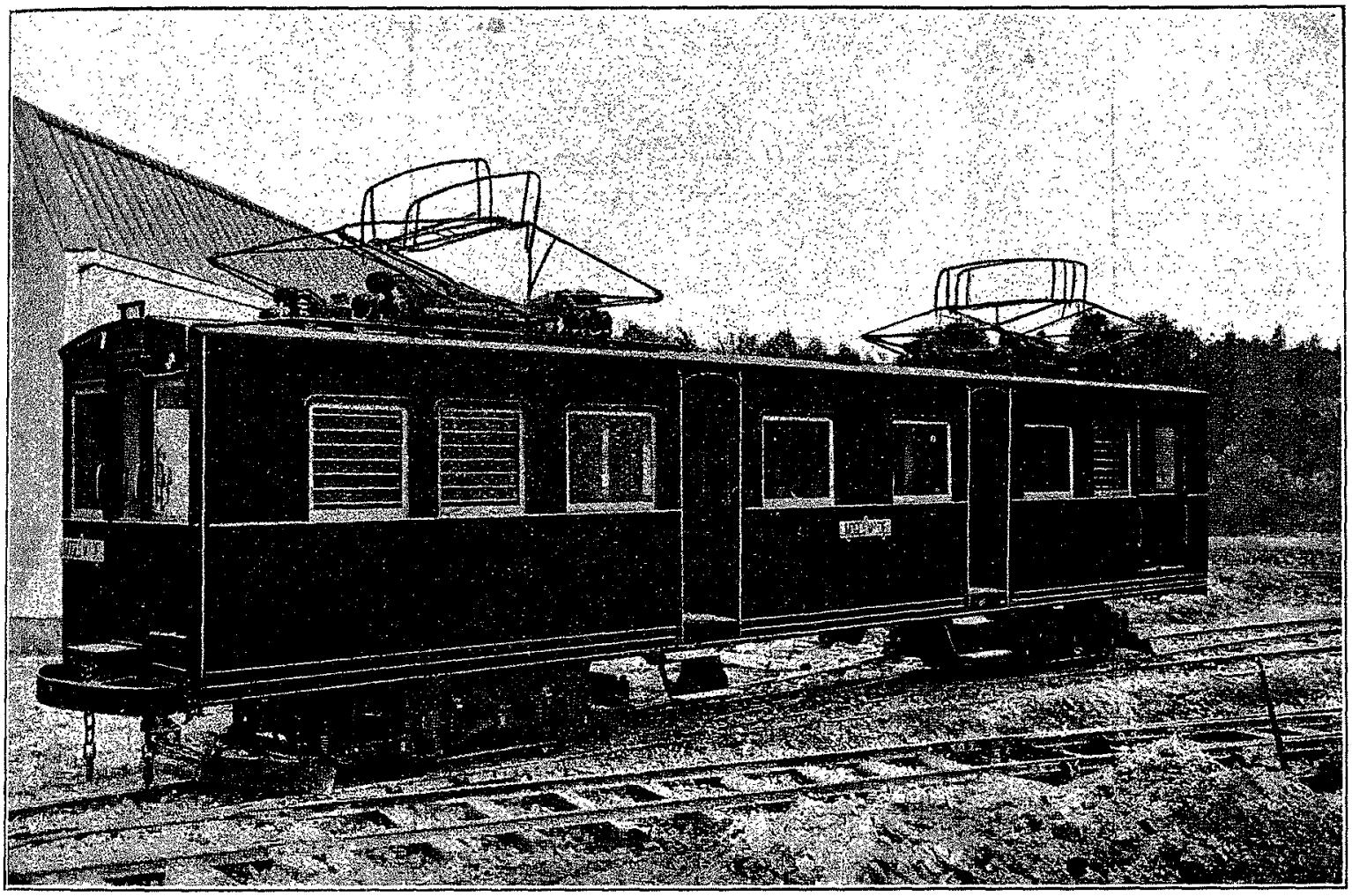

FIG. 9. - VuE D'UNE AUTOMOTRICE A Boggie se jette dans la Mohaw River. Celle nouvelle usine. jointe à une ancienne usine hydro-électrique,également située sur l'East Canadn Creek, à 6 kilomètres plus bas, et à une usine à vapeur de secours de $100 \mathrm{HP}$, située à Tribes Hill, à 50 kilomètres de là, doit fournir l'énergie électrique nécessaire à la traction des trains du Fonda, Johnston d: Gloversville Railway, et à assurer en même temps un service public de distribution électrique dans toute la rógion avoisinante ( $\mathrm{T}$ )

La nouvelle installation comprend un barrage, un? conduite forcée de rọo mètres de longueur, et unc usine génératrice. Elle a été prévue pour pouvoir dévelopner ulférieurement I 2 oon HP. mais. à l'heure actuelle, 8 ono HP senle-

nent soit par action direcle de l'air sur les cylindres des freins, soit par dépression, comme dans le cas général du frein automatique. Il existe en outre un frein de secours à main. Enfin, en cas de danger, on obtiendrait encore un freinage énergique en faisant fonctionner les moteurs sur lis manche arrière.

l'air comprimé nécessaire à la manœuvre de l'archet et i la commande des freins cst oblenu par un compresseur d'air qui est actionné par un moteur sćrie fonctionnant sous yoo volts. Ce moteur est mis en marche ou à l'arrêt par un régulateur automatique qui maintient la pression entre 5 ct 7 kilogs par centimètre carré. Si par suile d'un arrêt prolongé, ou pour toute autre raison, la pression venait à faire défaut, l'archet peut être appliqué contre le fil de contact au moyen d'une pompe à main agissant sur son circuit d'air comprimé.

La traction électrique des trains el des tramways par courant alternatif monophasé se généralise de plus en plus, ment sont en service. En voici la description sommaire.

Barrage. - Ce barrage est établi en un point où la rivière a 45 mètres environ de largeur, et se trouve encaissée entre deux parois calcaires à pic. Il est en béton, et a une hauteur maxima de 37 mètres avec une longueur à la crête de 120 mètres. Il crée un réservoir de 5 millions de mètres cubcs.

Il est prolongé par un déversoir de superficie de 60 mètres de longueur, arrasé à $2^{\mathrm{m}} / 2$ en contrebas de la crête. En oulre, à 30 mètres au-dessous de cette crête, il est muni de deux vannes de fond commandant deux conduites en tôles d'acier de $\mathrm{I}^{\mathrm{m}} 83$ de diamètre. Entre le déversoir et ces vannes de fond, on peut évacuer ainsi jusqu'à 420 mètres cubes par seconde, alors que les plus fortes crues connues n'ont pas dépassé 3 ro mètres cubes.

Conduite. - La conduite forcéc actucllement installéc a rq̣ mètres de longucur. Elle est en tôle d'acier, de $n, 5$ à

(1) D'après l'Engineering Record du 8 juin Igı • 
I 2,7 millimètres d'épaisseur, et son diamètre intérieur est de $2^{m} 7 / 3$. Elle est supportée par des pilicrs en béton, puis recouverte de terre. Une couche de peinture de mininm et de plombagine la préserve contre la rouille. De plus, pour la raidir', on l'a munie de cornièces circulaires, de $\mathrm{r}_{27} \times 89 \times 9,5$ millimètres, disposées tous les $3^{\mathrm{m}} 66$.

A 127 mètres du barrage, on a disposé une cheminéc d'équilibre, de $6^{\mathrm{m}}$ Io de diamètre ef de 23 mètres de hauteur. Celte cheminće doit surtout servir à diminuer l'intensité des coups de bélier négatifs, car lout déversement y est impossible en cas de coup de bélier proprement dit, lc sommet do la cheminée étant à $6^{\mathrm{m}} 10$ au-dessus de la crète du barrage.

A son extrémité amont, la conduite débouche dans une chambre à deux compartiments, munis chacun d'une grille et d'une vanne. Fn tête de la conduite se trouve une vanne d'arrêl, puis, immédiatement à l'aval, un reniflard. A son arrivéc à l'usine, la conduile se subdivise en deux troncons,

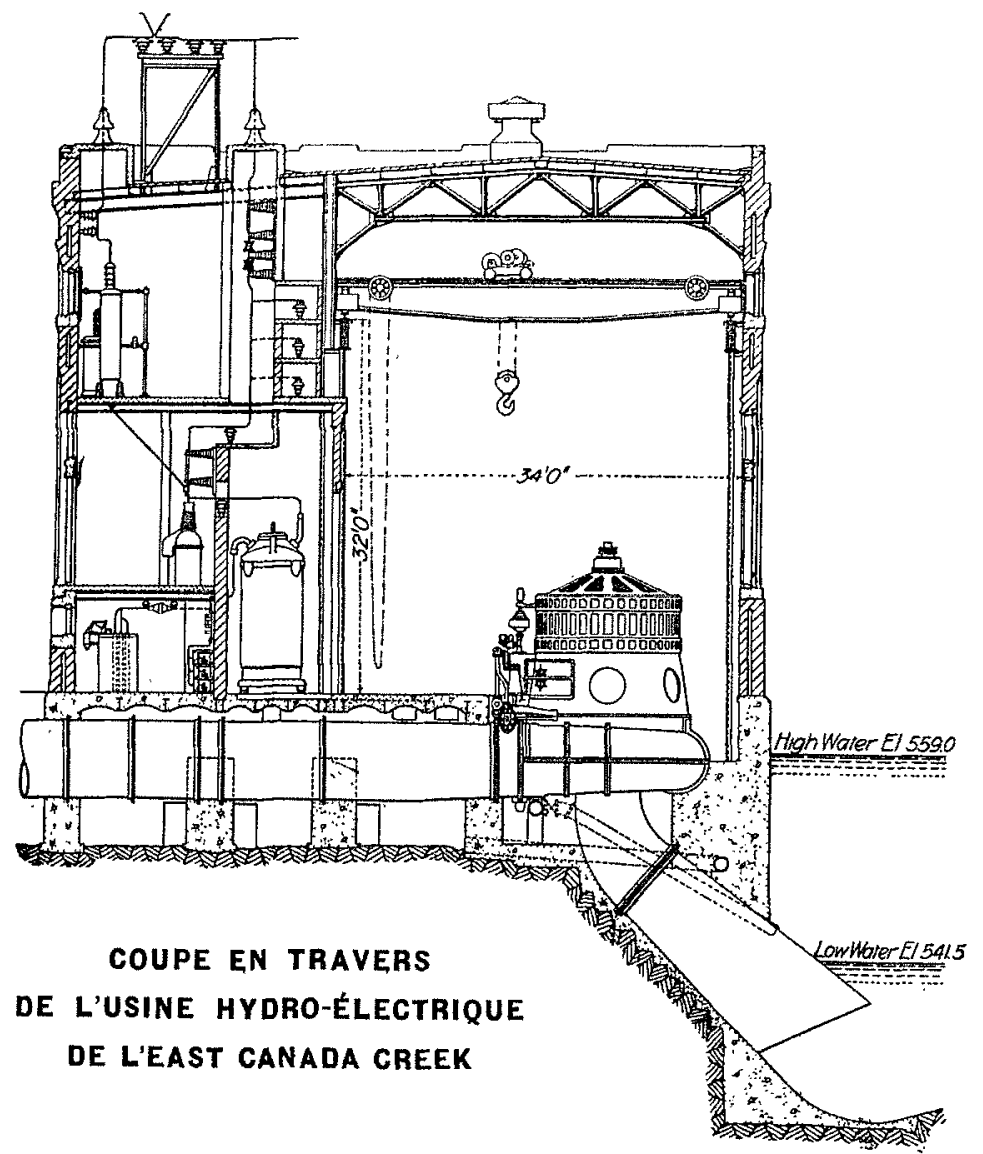

munis chacun d'unc vanne à papillon, el alimentant une turbine. Deux joints de dilatation ont été installés, l'un en avant, el l'aulre en aval de la chemince d'équilibre.

Ultérjeurement, on doit placer une scconde conduite, de ${ }^{\mathrm{m}} 9 \delta \mathrm{r}$ de diamètre.

Usine. - L'usine est construite en briques, avec piliers en acier. A l'heure actuelle, elle mesure en plan $23^{\mathrm{m}} 5$ o de long sur 17 mètres de large, et contient 2 groupes électrogènes à axe verlical de 1 ooo HP, avec leur apparcillage. U\}térieurement, elle sera agrandic pour recevoir un troisième groupe.

Les turbines sont du type Francis à aspiration, à hûche spiraloide en fonte. La couronne distributrice est composée de vanettes mobiles en acier forgé, et la rouc mobile est en bronze, de $\mathrm{I}^{\mathrm{m}_{22}}$ de diamètre. La puissance normale est de 4 ooo HP, el la hateur de chme est comprise entre $33^{\mathrm{m}} 7^{5}$ et 36 mètres suivant l'élat de la rivière. I a vilesse de rolation est de 3 oo toud's par minute. Le réglage de la vitesse est. obtenu au moyen d'un régulateur à servo-motemr à huile sous pression.

Ces turbines ont élé foumies par la Pelton Water Wheel Company, qui a garanti un rendement maximum de $8.1 \%$.

Chaque turbine est dircctement accouplée à un alternatcur de $2800 \mathrm{KVA}$, produisant du courant triphasé à 2800 volts et 25 périodes par seconde.

Deux transformateurs à bain d'huile, et refroidissement par circulation d'cau, élèvent la tension de 2300 à 30000 volts. Ils ont été prévus pour pouvoir élever ultérieurement cette tension à 60 ooo volts.

Le courant d'excitation est fourni par deux dynamos à courant continu, de 125 kilowatls, dont l'une est actionnée par une turbine horizontale Francis de $200 \mathrm{HP}$, qui reçoit l'eau par une dérivation faite sur le tuyau de l'une des grosses turbines verticales, et dont l'autre est actionnée par un moleur d'induction de 200 chevaux.

Tout le matériel électrique a été fourni par la General Electric Company.

De l'usine génératrice part une ligne à 30 ooo volts (plus lard à 60 noo), aboutissant à la slation de Tribes Ilill. Collo ligne, qui a 50 kilomètres de longueur, est supportée par des poteanx métalliques qui ont I 8 mètres de hauteur et sont rsparćs on moyonne do i6- mèlues.

Une ligne à 6 noo volts relie cette usine hydro-électrique avec l'ancienne usine d'aval. Flle fut élablie en premier lieu, d. manière à permeltre l'alimentalion en énergie élcctrique des chantiers de construction du barage, de la conduite of de l'usine, soil pour l'échairage, soil pour la forec molrice.

Comme cette ancienne usine hydro-électrique d'aval pro. duit du courant à 60 cycles, on a installé un changeur de fréquence de 500 kilowatts, permettant de passer de la fríquence 62 à la fréquence 25 , ou inversement. On a prévu la place nécessaire pour un second appareil de même puissance.

J. G.

\section{RÉSISTANCE DES MATÉRIAUX}

\section{TRAITEMENT PRÉALABLE DES TOLES AVANT LEUR EMPLOI EN CHAUDRONNERIE}

La question du meilleur métal à employer pour la construction des chaudières et des conduites forcées pour usines lydro-b́lechriques, afin d'éviler les fissures ou avaries diverses, se prósente actuellement cncore dans les mêmes conditions que depuis un ccrtain nombre d'années.

Les fournisseurs de tôles dégagent leur responsabilité en disant qu'ils livrent un métal excellent puisqu'il résiste victoricusement à tous les essais imposés par les cahicrs des charges rigoureux qui ont été établis par l'accord des Associations de surveillance des chaudières. Si donc il survient des avaries en service ou pendant la construction, cc n'est pas la qualilé du métal qu'il faut incriminer, mais bien de mauvais procédés de travail dans les ateliers de chatdronnerie.

A ces arguments des métallurgistes, les constructeurs peuvent répondre, avec quelque raison, qu'ils demandenit avant loul un métal moins délical à traiter, of capable de bien résister au travail forcément un peu brutal de la chaudronnerie, et aussi aux exigences de service des chaudières. 